\title{
Retrospective Assessment of Postoperative Nausea and Vomiting after Unrestricted Clear Fluids before Day Surgery: An Observational Study
}

\author{
Prabodh Bansal', Rakesh Roshan ${ }^{2}$ \\ ${ }^{1}$ Associate Professor, Department of Surgery, Rama Medical College Hospital \& Research Centre, Hapur, Uttar Pradesh, India, ${ }^{2}$ Senior Resident, Department of \\ Surgery, Rama Medical College Hospital \& Research Centre, Hapur, Uttar Pradesh, India.
}

\section{Abstract}

Background: Nausea and vomiting during and after the surgery are distressing for both the patient and his/her family. In addition, when vomiting occurs intraoperatively under regional anaesthesia, it offers significant challenge to the operating surgeon, potentially increasing the duration of the procedure and the risk of bleeding and inadvertent associated surgical trauma. Aim of the study: To assess postoperative nausea and vomiting after unrestricted clear fluids before day surgery. Subjects and Methods: The study was conducted in the Department of Surgery, Rama Medical College Hospital \& Research Centre, Hapur, Uttar Pradesh, India. For the study, medical records of 200 patients who underwent surgical procedure at the hospital were selected for review. It was made sure that included patients were restricted from fluid intake for 12 hours before the surgery. We analysed records of randomly selected 200 patients who underwent surgical procedure at the hospital for the study period. Results: A total of 200 patient records randomly selected from the pool of records were analysed in the present study. We observed that mild nausea was experienced by 27 patients, moderate was experienced by 16 patients and severe nausea was experienced by 3 patients. We observed that 14 patients had vomiting only once, 8 patients had 2-3 times vomiting and 2 patients had vomiting more than 3 times. The results were compared and found to be statistically non-significant. Conclusion: From the results of the present study, this can be concluded that restriction of food and fluid intake before the day surgery is an efficacious method in avoiding nausea and vomiting in patients during post-operative time.

Keywords: Nausea, Vomiting, Surgery.

Corresponding Author: Dr. Prabodh Bansal, Associate Professor, Department of Surgery, Rama Medical College Hospital \& Research Centre, Hapur, Uttar Pradesh, India.

Received: July 2019

Accepted: July 2019

\section{Introduction}

Pre-operative fasting, defined as the restriction of food and fluid intake prior to general anaesthesia or sedation, is vital for patient safety. Induction of anaesthesia or sedation results in a depression of the gag, cough and swallow reflexes that normally protect the airway, placing patients at risk of pulmonary aspiration, pneumonia and even death should regurgitation or vomiting of gastric contents occur. ${ }^{[1]}$ Therefore, for many years pre-operative patients were restricted from food and drink for periods of eight to 12 hours before induction of anaesthesia or sedation. Nausea and vomiting are common symptoms experienced during surgery under regional anaesthesia and can also occur in the postoperative period under either regional or general anaesthesia. ${ }^{[2,3]}$ Nausea and vomiting during and after the surgery are distressing for both the patient and his/her family. In addition, when vomiting occurs intraoperatively under regional anaesthesia, it offers significant challenge to the operating surgeon, potentially increasing the duration of the procedure and the risk of bleeding and inadvertent associated surgical trauma. ${ }^{[4]}$ There is also the potential risk of aspiration of gastric contents when vomiting occurs intraoperatively. There are multiple underlying causes of nausea and vomiting at surgery and several different modes of intervention are in common use. Many different studies have evaluated these interventions, either compared with one another or with placebo. In some countries, for example in the $\mathrm{UK}$, there is a recommendation for the routine administration of drugs (antiemetics) or acupressure to try to prevent nausea and vomiting at surgical table. ${ }^{[5]}$ However, it is probably more common clinical practice to give antiemetic medication as treatment, rather than as prophylaxis. It is not known to what extent medications which are efficacious as treatment are also efficacious as prophylaxis (and vice versa). ${ }^{[6]}$ The arguments against prolonged fasting are no less significant. More aggressive surgeons tend to reduce the hours by allowing clear liquid's till as late as 2 hours before induction. They bring the findings of jujunal mucosal atrophy starting as early as 6 hours after last fluid intake, leading to transmucosal migration of organisms, especially in compromised patient. Others however promulgate the that fasting being one of the stressors leads to generalized failure to copeup with surgical stress, inturn leading to increased complication rate of all types. Yet others profound that oral and gastric secretion of 
1-2 liters in stomach at the time of induction can cause all the damage ascribed to intra operative vomiting.

In an effort to settle this big question- to fast or not to fast this study was undertaken with comparison from other studies in literature.

\section{Subjects and Methods}

The study was conducted in the Department of Surgery, Rama Medical College Hospital \& Research Centre, Hapur, Uttar Pradesh, India. The ethical clearance for study protocol was obtained from ethical committee of the institution. For the study, medical records of 200 patients who underwent surgical procedure at the hospital were selected for review. It was made sure that included patients were restricted from fluid intake for 12 hours before the surgery. According to hospital policy, the patients are called by nurses for follow-up after discharge and to assess various domains such as their general feeling post-operatively, experience of dizziness, nausea (mild, moderate, severe), vomiting (once, 2-3 times, $>3$ times), pain (mild, moderate, severe), and bleeding. We analysed records of randomly selected 200 patients who underwent surgical procedure at the hospital for the study period.

The statistical analysis of the data was done using SPSS version 11.0 for windows. Chi-square and Student's t-test were used for checking the significance of the data. A pvalue of 0.05 and lesser was defined to be statistical significant.

\section{Results}

Table 1: Number of patients with nausea post-operatively.

\begin{tabular}{|l|l|l|l|}
\hline \multirow{2}{*}{} & \multicolumn{3}{|l|}{ Nausea } \\
\cline { 2 - 4 } & Mild & Moderate & Severe \\
\hline Number of patients & 27 & 16 & 3 \\
\hline Total no. of patients & 200 & \multicolumn{2}{|l}{} \\
\hline
\end{tabular}

Table 2: Number of patients with vomiting post-operatively

\begin{tabular}{|l|l|l|l|}
\hline \multirow{2}{*}{} & Vomiting & \multicolumn{3}{|c|}{-3 times } \\
\cline { 2 - 4 } & Once & $\mathbf{2 - 3}$ times & 2 \\
\hline Number of patients & 14 & 8 & \multicolumn{2}{|l}{} \\
\hline Total no. of patients & 200 &
\end{tabular}

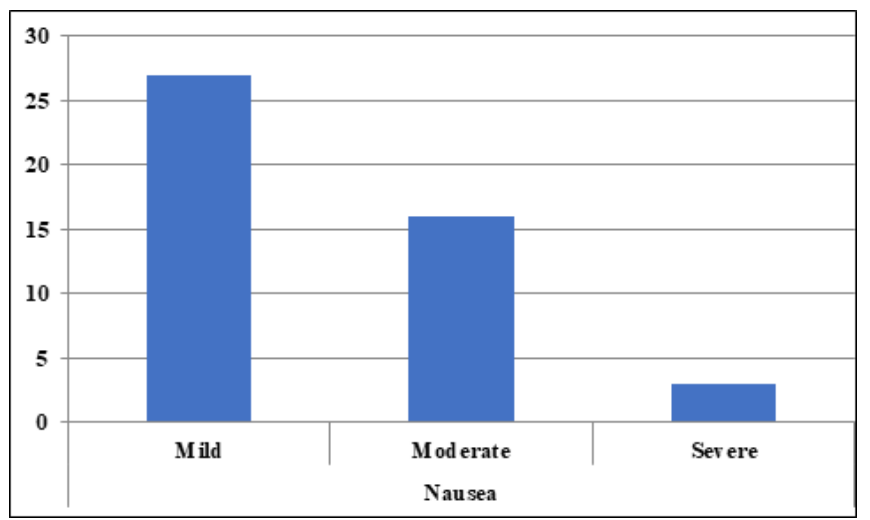

Figure 1: Post-operative Nausea in patients

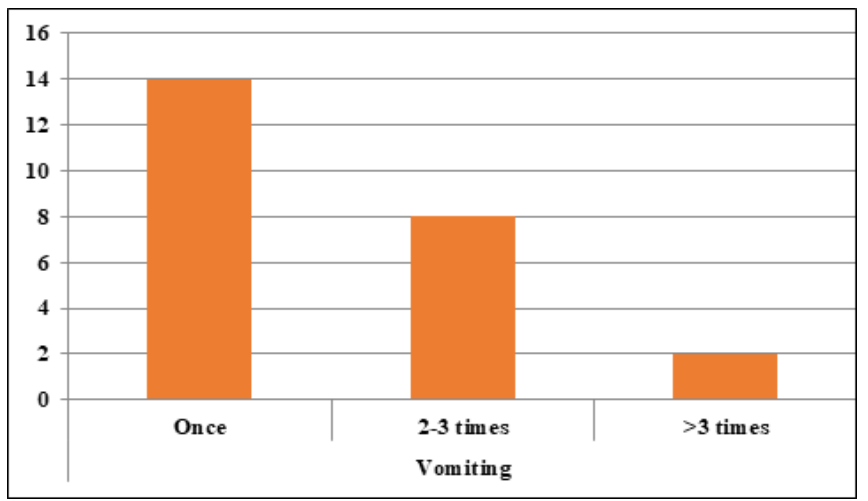

Figure 2: Post-operative vomiting in patients

A total of 200 patient records randomly selected from the pool of records were analysed in the present study. [Table 1] shows the number of patients with nausea postoperatively. We observed that mild nausea was experienced by 27 patients, moderate was experienced by 16 patients and severe nausea was experienced by 3 patients [Figure 1]. [Table 2] shows the number of patients with vomiting postoperatively. We observed that 14 patients had vomiting only once, 8 patients had 2-3 times vomiting and 2 patients had vomiting more than 3 times [Figure 2]. The results were compared and found to be statistically non-significant.

\section{Discussion}

In the present study, 200 patients' records were assessed. It was observed that the incidence of nausea and vomiting after the procedure was less. However, nausea was more common than vomiting. Most commonly patients experienced mild nausea and vomiting only one time. The results were found to be statistically non-significant. The results were compared to previous studies in the literature. Abdullah Al Maqbali $\mathrm{M}$ et al assessed the duration of fasting before elective surgery on the part of adult patients. A prospective study was conducted to identify the fasting time and complications among surgical patients undergoing elective surgery over a 4-month period. The patients were asked for preoperative fasting times, and the complication. The demographic data were taken from the patients' files. A total of 169 patients were included in the study, 88 male and 81 female. The minimum and maximum fasting hours with regard to food were 7 hours and 19 hours, respectively; all the patients fasted from food for longer than the recommended time. The minimum and maximum fasting hours for fluids were 4 hours and 19 hours, respectively; all the patients fasted from fluid for longer than the recommended time. They concluded that most of the patients fasted from food and fluids for more than the time recommended by the American Society of Anaesthesiologists, the Royal College of Nursing, the Association of Anaesthetists of Great Britain and Ireland and the Royal College of Anaesthetists. Excessive fasting could lead to discomfort and possible morbidity in surgical patients. The surgical team needs to collaborate to reduce the fasting time by revising the operative list. Ingadottir B et 
al explored how long patients fast before surgery and what instructions they received, one year after the introduction of new guidelines for patients and professionals. This descriptive study was undertaken in a national, 660-bed university hospital in 2011. Data was collected from patient records and with questionnaires. Included were adult surgical patients having anaesthesia during a 5day period. The sample consisted of 193 patients: $83 \%$ were scheduled for elective surgery and $86 \%$ returned questionnaires. Average fasting time was $13,6( \pm 3.0)$ hours for solid food and 8,8 $( \pm 4.5)$ hours for clear fluids. A quarter $(27 \%)$ had received instructions according to guidelines and $45 \%$ were instructed to fast from midnight. Information was either written $(18 \%)$, verbal $(37 \%)$ or both $(45 \%)$ and $46 \%$ of patients received information on the importance of fasting. Patients scheduled for morning surgery fasted for a shorter time than afternoon patients $(\mathrm{p}<0.05)$. Patients who received both verbal and written information fasted shorter on clear fluids $(p<0.001)$ than others. They concluded that the fasting of surgical patients before their operation is unnecessarily long and they do not get uniform instructions. This warrants further exploration. There is a need for staff to coordinate instructional practices, to involve patients more in their own care with consistent information and comprehensive education and assist them in reducing fasting on clear fluids after hospital admission. ${ }^{[7,8]}$ In general a tendency was found in patients to prolong and embolden the fasting thinking that a better procedure will result from longer fasting.

Klemetti $\mathrm{S}$ et al examined whether active counseling and more liberal oral fluid intake decrease postoperative pain, nausea and vomiting in pediatric ambulatory tonsillectomy. Families, whose child was admitted for ambulatory tonsillectomy or adenotonsillectomy, were randomly assigned to the study groups $(n=116 ; 58$ families in each group). The intervention group received the fasting instructions with face-to-face counseling for the child's active preoperative nutrition, and the control group the fasting instructions according to the hospital's standard procedure. The level of postoperative pain and nausea was scored in the postanesthesia care unit (PACU) during the first postoperative hour, as well as at, ${ }^{[2,4,8]}$ and $24 \mathrm{~h}$ postoperatively. The first scoring in PACU was performed by the attending nurse with a 0-10 scale. The rest of the estimations were made independently and simultaneously by the children using a VAS scale, and by the parents using a 0-10 scale. The children in the control group were in more pain in the PACU than the children in the intervention group, and the difference between the groups was statistically significant $(\mathrm{p}=0.0002)$. All pain scores, according to the children and the parents, increased after the surgery. In both groups the highest score values were found at home $8 \mathrm{~h}$ after surgery, and no significant difference was found between the study groups. On the first postoperative morning, the children in the control group were in pain $(\mathrm{p}=0.047)$. The children did not have significant nausea in the PACU, but the nausea increased postoperatively. Four hours after surgery the children were most nauseous according to all estimations $(60 \%, \mathrm{n}=116)$. More than half of the children vomited and most vomited clotted blood. Nausea and vomiting decreased during the evening of the surgery, but six children vomited the next morning, four of them vomited blood. The incidence and intensity of postoperative nausea and vomiting between the intervention and control groups were not statistically significant. However, preoperative nutritional counseling and more liberal per oral fluid intake appeared to have a positive effect on the children's well-being and helped them to better tolerate postoperative nausea and vomiting. They concluded that the preoperative counseling about active preoperative nutrition significantly reduces the child's pain during the first posttonsillectomy hours and might prepare the child to better tolerate the stress of potential postoperative nausea and vomiting. Järvelä $\mathrm{K}$ et al evaluated the effect of a preoperative oral carbohydrate drink vs. overnight fasting on perioperative insulin requirements in non-diabetic patients undergoing elective coronary artery bypass grafting (CABG) surgery. One hundred and one patients scheduled for $\mathrm{CABG}$ were enrolled in the study. After fasting overnight, the patients were randomised into two groups. In the control group (C), no drink was given in the morning. In the treatment group (T), the patients ingested $400 \mathrm{ml}$ of carbohydrate fluid $2 \mathrm{~h}$ before induction of anaesthesia. Blood glucose and insulin requirement was recorded. Gastric drainage was measured. Post-operative nausea and vomiting was recorded. Neither the number of patients requiring insulin nor the amount of insulin required to maintain normoglycaemia differed between the study groups. More patients in the treatment group experienced nausea post-operatively ( 26 vs. $16, \mathrm{P}=0.044$ ), but vomiting was equally common in the study groups (10 vs. 7). Intraoperative gastric drainage was $26.8+/-57.9 \mathrm{ml}$ in the treatment group vs. $16+/-37.9 \mathrm{ml}$ in the control group (NS). They concluded that patient population, a pre-operative oral carbohydrate drink did not reduce post-operative insulin resistance or post-operative nausea and vomiting. According to findings, it is safe to allow cardiac surgery patients to drink clear fluids up to $2 \mathrm{~h}$ before induction of anaesthesia, because gastric emptying of the drink was almost total and no aspiration occurred..$^{[9,10]}$

\section{Conclusion}

From the results of the present study, this can be concluded that restriction of food and fluid intake before the day surgery is an efficacious method in avoiding nausea and vomiting in patients during post-operative time. As regard the complication and other stress related consequences of preoperative fasting on result of operative procedures another long term study shall be undertaken and be reported through this channel.

\section{References}

1. Brady M, Kinn S, Stuart P. Preoperative fasting for adults to prevent perioperative complications. Cochrane Database of Systematic Reviews 2003;(4):CD004423.

2. Perioperative fasting in adults and children - a RCN guideline for the 
multidisciplinary team. Clinical practice guidelines, RCN publications, 2005.

3. American Society of Anaesthesiologist task force on preoperative fasting. Practice guidelines for preoperative fasting and the use of pharmacologic agents to reduce the risk of pulmonary aspiration: application to healthy patients undergoing elective procedures: an updated report by the American Society of Anaesthesiologists Committee on standards and practice parameters. Anaesthesiology 2011;114(3)495-511.

4. Gilbert SS, Easy WR, Fitch WW. The effect of preoperative oral fluids on morbidity following anaesthesia for minor surgery. Anaesthesia 1995; 50:79-81.

5. Agarwal A, Chari P, Singh H. Fluid deprivation before operation. The effect of a small drink. Anaesthesia 1989; 44:632-34.

6. Goodwin APL, Rowe WL, Ogg TW, et al. Oral fluids prior to day surgery. The effect of shortening the preoperative fluid fast on postoperative morbidity. Anaesthesia 1991; 46:1066-1068.

7. Abdullah $\mathrm{Al}$ Maqbali M. Preoperative fasting for elective surgery in a regional hospital in Oman. Br J Nurs. 2016 Jul 28;25(14):798-802. doi: 10.12968/bjon.2016.25.14.798.

8. Ingadottir B, Olafsdottir AM, Sveinsdottir H, Asmundsdottir LB, Asgeirsdottir L, Torp MS, Hafsteinsdottir EJ. Preoperative fasting: Instructions to patients and length of fasting - a prospective, descriptive survey. Laeknabladid. 2016 Jun;102(6):283-8. doi: 10.17992/lbl.2016.06.86.

9. Klemetti S, Kinnunen I, Suominen T, Antila H, Vahlberg T, Grenman $\mathrm{R}$, Leino-Kilpi $\mathrm{H}$. The effect of preoperative fasting on postoperative pain, nausea and vomiting in pediatric ambulatory tonsillectomy. Int J Pediatr Otorhinolaryngol. 2009 Feb;73(2):263-73. doi: 10.1016/j.ijporl.2008.10.014. Epub 2008 Dec 4.

10. Järvelä K, Maaranen P, Sisto T. Pre-operative oral carbohydrate treatment before coronary artery bypass surgery. Acta Anaesthesiol Scand. 2008 Jul;52(6):793-7. doi: 10.1111/j.1399-6576.2008.01660.x. Epub 2008 May 12.

Copyright: (C) the author(s), 2019. It is an open-access article distributed under the terms of the Creative Commons Attribution License (CC BY 4.0), which permits authors to retain ownership of the copyright for their content, and allow anyone to download, reuse, reprint, modify, distribute and/or copy the content as long as the original authors and source are cited.

How to cite this article: Bansal P, Roshan R. Retrospective Assessment of Postoperative Nausea and Vomiting after Unrestricted Clear Fluids before Day Surgery: An Observational Study. Asian J. Med. Res. 2019;8(3):SG04-SG07.

DOI: dx.doi.org/10.21276/ajmr.2019.8.3.SG2

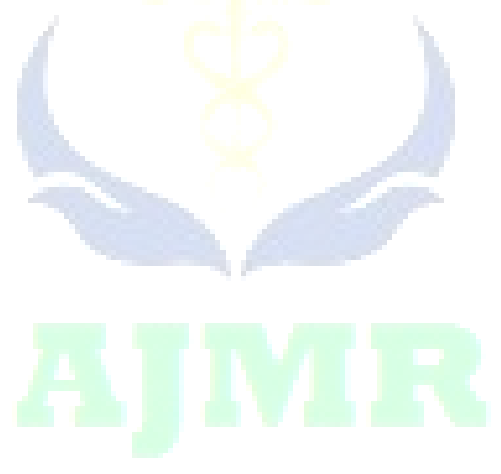

